\title{
"Micro Hand S" Surgical Robot vs Laparoscopic Gastrectomy for Gastric Cancer: Comparison of Short-Term Outcomes
}

\section{Yuan-Bing Yao}

Central South University Third Xiangya Hospital https://orcid.org/0000-0002-2221-5745

\section{Guo-Hui Wang}

Central South University Third Xiangya Hospital

\section{Yong Liu}

Central South University Third Xiangya Hospital

\section{Bo Yi}

Central South University Third Xiangya Hospital

\section{Zheng Li}

Central South University Third Xiangya Hospital

Shai-Hong Zhu ( $D$ zshcsu@163.com)

https://orcid.org/0000-0003-2496-9417

\section{Research article}

Keywords: Surgical robot, Gastric cancer, Gastrectomy, Safety, Feasibility

Posted Date: July 15th, 2020

DOl: https://doi.org/10.21203/rs.3.rs-41120/v1

License: (1) This work is licensed under a Creative Commons Attribution 4.0 International License. Read Full License 


\title{
"Micro Hand S" surgical robot vs laparoscopic gastrectomy for gastric cancer: comparison of short-term outcomes
}

\section{Running head: "Micro Hand S" robot vs laparoscopic gastrectomy}

\author{
Yuan-Bing Yao', Guo-Hui Wang ${ }^{1}$, Yong Liu', Bo Yi', Zheng Li ${ }^{1 *}$, Shai-Hong Zhu' ${ }^{1}$ * \\ * Corresponding Author \\ Shai-Hong Zhu \\ zshcsu@163.com 18975186193 \\ Zheng Li \\ swiftjeff@163.com 13347320595
}

\begin{abstract}
Background Expensive cost of surgery has limited the use of surgical robot in China. The emergence of "Micro Hand S" surgical robot provides more choices for surgical treatment. Our study was to evaluate the safety and feasibility of "Micro Hand S" surgical robotic gastrectomy for gastric cancer.

Methods Perioperative data of 75 patients who underwent "Micro Hand S" surgical robotic gastrectomy (RG) or laparoscopic gastrectomy (LG) at the Department of General Surgery, Third Xiangya Hospital, Central South University from June 2017 to January 2019 were collected to compare the short-term outcomes between the two groups.

Results No statistically significant difference was found in baseline characteristics between the two groups $(\mathrm{P}>0.05)$. RG group had longer operation time $(\mathrm{P}<0.01)$. The harvested lymph nodes were comparable between the two groups $(\mathrm{P}=0.084)$. There were five positive margins in the LG group, and the $\mathrm{R} 0$ resection rate was comparable between the two groups $(\mathrm{P}=0.247)$. 16 complications occurred in the RG group according to the Clavien-Dindo classification system, 6 in grade I, and 10 in grade II. 68 complications happened in the LG group, 22 in grade I, 44 in grade II, 1 in grade IIIa and 1 in grade IVa. The comprehensive complications index $(\mathrm{CCI})$ was similar between the two groups $(\mathrm{P}=0.895)$. Intraoperative blood loss, surgical resection, reconstruction of the digestive tract intraoperative blood transfusion, Chemoradiotherapy, pathological type, degree of differentiation, proximal resection margin, time of first flatus, time of liquid diet, time of abdominal drainage tube extraction, and hospital stay for RG and LG were similar too $(\mathrm{P}>0.05)$.
\end{abstract}

Conclusion "Micro Hand S" surgical robotic gastrectomy is safe and feasible.

Keywords Surgical robot; Gastric cancer; Gastrectomy; Safety; Feasibility

\section{Introduction}

Gastric cancer, the fourth most common malignant tumor in the world, is also the second 
leading cause of death for all malignant tumors [1]. In China, gastric cancer ranks third in morbidity and mortality, causing a heavy disease burden [2]. Surgical operation is the main treatment for gastric cancer. Gastrectomy with lymph node dissection is the gold standard for the treatment of gastric cancer, and laparoscopic surgery has been the most commonly used surgical approach at present [3]. However, the laparoscopic approach has obvious limitations, such as two-dimensional vision, hand tremor amplification, limited range of motion, and uncomfortable posture [4]. Surgical robots - the inevitable outcome of the development of minimally invasive surgery-have some special advantages that laparoscopes do not have, including 3-dimensional amplificated vision, tremor filtering, motion scaling, and comfortable posture [5]. Since Hashizume et al [6] first reported the da Vinci gastrectomy in 2002, researches on robotic gastrectomy have increased [7-9]. In January 2019, the "Micro Hand S" surgical robot [10], independently developed in China for more possibilities of surgical treatment, successfully completed the phase I clinical trial. In this study, we compared the short-term outcomes between "Micro Hand S" surgical robotic gastrectomy (RG) and laparoscopic gastrectomy (LG) through retrospective analysis for evaluating the safety and feasibility of the robotic gastrectomy.

\section{Materials and methods}

\section{Patients}

We retrospectively collected the perioperative data of 75 patients who underwent RG or LG at the Department of General Surgery, Third Xiangya Hospital, Central South University from June 2017 to January 2019. There were 15 cases in the RG group and 60 cases in the LG group. Inclusion criteria were as follows: (1) complete perioperative data could be obtained; and (2) 
biopsy confirmation of gastric cancer. Exclusion criteria were as follows: (1) metastasis or complicated with other tumors; (2) gastric stump carcinoma or benign tumor; (3) leiomyoma or stromal tumor; (4) serious cardiopulmonary dysfunction or liver and kidney dysfunction; and (5) palliative operation. Preoperative examinations including blood and urine (routine examination), stool (routine examination), liver and kidney function, and blood coagulation function, along with electrocardiograms, investigation of tumor markers, ultrasound, computed tomography (CT) scans, and biopsy were performed to confirm the diagnosis. Patients were well informed of the surgical procedure and risks, complications, alternative treatments, and voluntarily signed informed consent. After the operation, patients were treated with gastrointestinal protective agents, antibiotics treatment, fluid infusion, nutritional support, maintenance of electrolyte balance, and other symptomatic treatments. Chemoradiotherapy was performed in line with Japanese gastric cancer treatment guidelines [11]. Our study was approved by the Institutional Review Board of the Third Xiangya Hospital and registered at ClinicalTrials.gov (NCT02752698).

\section{Surgical procedure}

In addition to trocar location and surgical instruments used, there was no significant difference between the RG and LG procedures for gastric cancer. Patients were first placed in the supine position; this was followed by routine disinfection and sterile drapes whisked onto the patients after general anesthesia. Pneumoperitoneum was established by puncture with a pneumoperitoneum needle under the umbilicus. Small incisions were made in the umbilicus, left lower abdomen, right lower abdomen, intersection of the umbilical plane, and the anterior axillary line to insert the trocars. The trocar placement and operation of the domestic robot are 
shown in Fig. 1. The exact locations of the trocars were as follows: A: $3-5 \mathrm{~cm}$ at the lower edge of the umbilical foramen, placed in a three-dimensional laparoscope; B: 5-8 cm below the costal margin of the left anterior axillary line, connected to the right robotic arm; C: 5-8 cm below the costal margin of the right anterior axillary line, connected to the left robotic arm; D: the intersection of the left midclavicular line and the umbilical plane, the auxiliary operation hole; E: the intersection of the right midclavicular line and the umbilical plane, the auxiliary operation hole. The gastrectomy and the extent of lymph node dissection were performed according to Japanese gastric cancer treatment guidelines. Reconstruction of domestic robots was performed in vitro through an approximately $10 \mathrm{~cm}$ incision. Roux-en-Y esophagojejunostomy was performed after total gastrectomy and Billroth II gastrojejunostomy was performed after distal gastrectomy.

\section{Perioperative data}

Baseline characteristics of patients, surgical factors, pathological parameters, and postoperative outcomes were collected. Baseline characteristics included age, body mass index (BMI), gender, American Society of Anesthesiologists (ASA) physical status classification, tumor diameter, tumor location, clinical TNM stage and pathological TNM stage. Surgical factors included operative time, intraoperative blood loss, gastric resection, reconstruction, intraoperative complications, intraoperative blood transfusion and Chemoradiotherapy. Pathological parameters included pathological type, histological type, Lauren's classification, proximal resection margin, R0 resection rate, and harvested lymph nodes. Postoperative outcomes included time of first flatus, time of liquid diet, time of abdominal drainage tube extraction, duration of hospital stay, comprehensive complications index (CCI), and Death 
within 90 days after surgery.

The clinical and pathological TNM stage referred to 8th edition of the AJCC TNM Staging System for Gastric Cancer [12]. CCI, a newly developed measurement method for complications, was calculated through $\mathrm{http}: / /$ cci.assessurgery.com online tools [13]. It integrates all recorded complications based on the Clavien-Dindo Complications Classification System [14] into a formula that is weighted by severity, ranging from 0 (no complications) to 100 (death). Comprehensive statistics of complications from mild to severe and quantification of results enable CCI to intuitively reflect the severity of postoperative complications.

\section{Statistical Analysis}

IBM SPSS Statistics version 20.0 was used for statistical analysis. Continuous variables were expressed as mean \pm standard deviation (SD), and an independent sample $t$-test or Wilcoxon rank sum test were used. Categorical variables were compared through the Pearson $\chi^{2}$ test or the Fisher's exact test. The Wilcoxon rank sum test was used for ordinal categorical variable. All statistical analyses were two-sided, and P values less than 0.05 were considered statistically significant. 

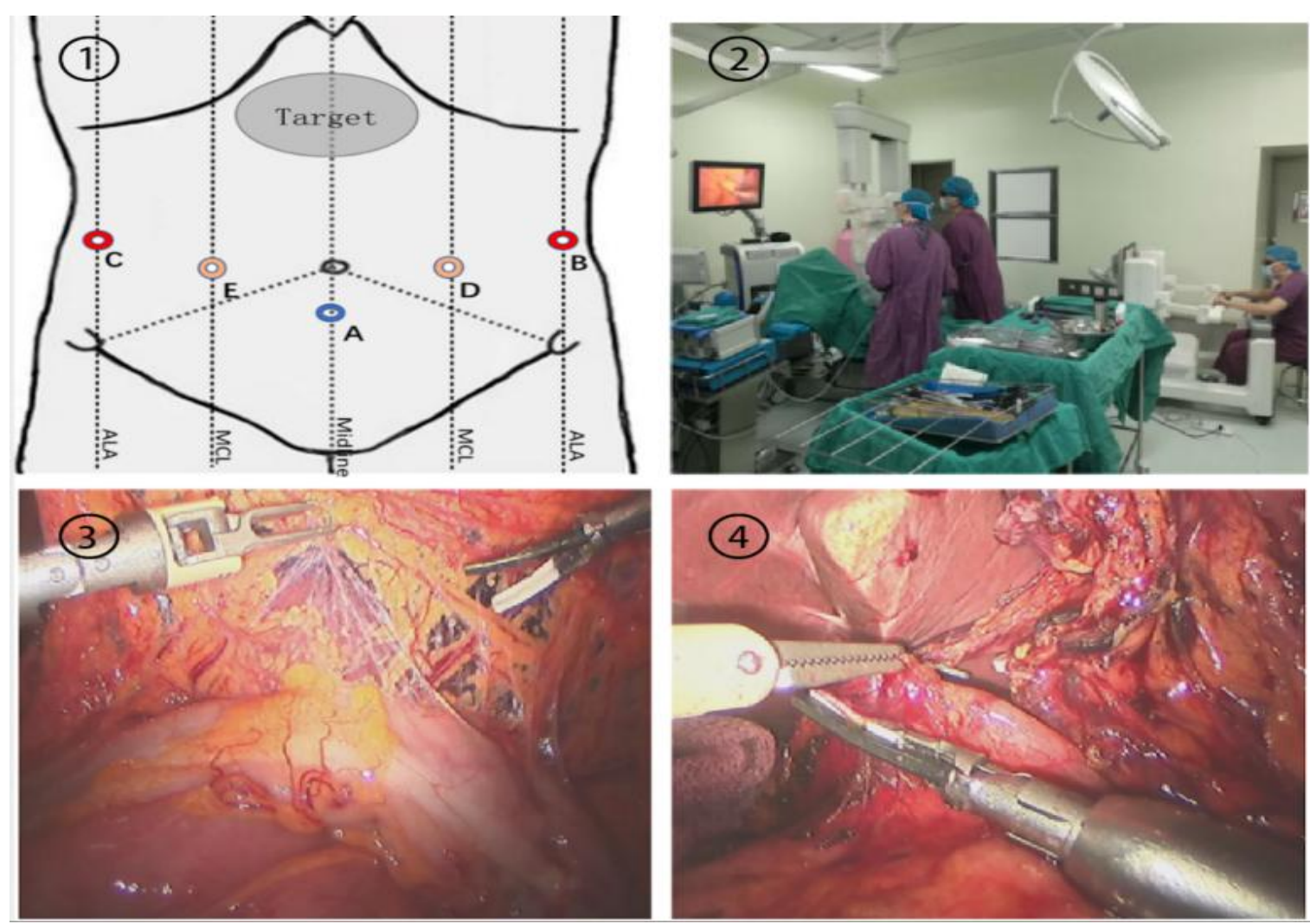

Fig.1 Trocar placement and operation of the domestic robot. (1) Trocar position. (2) The operating room. (3) Separating gastrocolic ligament. (4) Freeing common hepatic artery.

\section{Results}

\section{Baseline characteristics of patients}

Baseline characteristics of patients are shown in Table 1. The mean age of patients in the RG group was $61.7 \pm 7.4$ years and that in the $L G$ group was $57.0 \pm 12.3$ years $(\mathrm{P}=0.174)$. There were no significant differences in BMI, gender, ASA grade, tumor diameter, tumor location, clinical TNM stage or pathological TNM stage between the two groups $(\mathrm{P}>0.05)$.

Table 1 Baseline characteristics of patients with gastric cancer

\begin{tabular}{llll}
\hline & RG $(\mathrm{n}=15)$ & $\mathrm{LG}(\mathrm{n}=60)$ & $P$ value \\
\hline Age $($ years $)$ & $61.7 \pm 7.4$ & $57.0 \pm 12.3$ & 0.174 \\
BMI $\left(\mathrm{kg} / \mathrm{m}^{2}\right)$ & $21.6 \pm 3.2$ & $21.9 \pm 3.0$ & 0.815 \\
Gender & & & $>0.999$ \\
$\quad$ Male & 10 & 40 & \\
Female & 5 & 20 & \\
\hline
\end{tabular}




\begin{tabular}{|c|c|c|c|}
\hline ASA & & & 0.081 \\
\hline I & $0(0 \%)$ & $1(1.7 \%)$ & \\
\hline II & $4(26.7 \%)$ & $30(50.0 \%)$ & \\
\hline III & $11(73.3 \%)$ & $29(48.3 \%)$ & \\
\hline Tumor diameter $(\mathrm{cm})$ & $3.7 \pm 1.6$ & $3.8 \pm 1.6$ & 0.853 \\
\hline Tumor location & & & 0.618 \\
\hline Upper third & $1(6.7 \%)$ & $2(3.3 \%)$ & \\
\hline Middle third & $2(13.3 \%)$ & $12(20.0 \%)$ & \\
\hline Lower third & $12(80.0 \%)$ & $42(70.0 \%)$ & \\
\hline$\geq 2$ areas & $0(0.0 \%)$ & $4(6.7 \%)$ & \\
\hline cTNM stage & & & 0.898 \\
\hline I & $7(46.7 \%)$ & $13(21.7 \%)$ & \\
\hline II A & $0(0.0 \%)$ & $17(28.3 \%)$ & \\
\hline II B & $3(20.0 \%)$ & $10(16.7 \%)$ & \\
\hline III & $2(13.3 \%)$ & $10(16.7 \%)$ & \\
\hline IV A & $3(20.0 \%)$ & $6(10.0 \%)$ & \\
\hline IV B & $0(0.0 \%)$ & $0(0.0 \%)$ & \\
\hline TxNxM0 & $0(0.0 \%)$ & $4(6.7 \%)$ & \\
\hline $\mathrm{pT}$ & & & 0.165 \\
\hline $\mathrm{T} 1$ & $3(20.0 \%)$ & $15(25.0 \%)$ & \\
\hline $\mathrm{T} 2$ & $1(6.7 \%)$ & $5(8.3 \%)$ & \\
\hline $\mathrm{T} 3$ & $4(26.7 \%)$ & $24(40.0 \%)$ & \\
\hline $\mathrm{T} 4 \mathrm{a}$ & $2(13.3 \%)$ & $10(16.7 \%)$ & \\
\hline $\mathrm{T} 4 \mathrm{~b}$ & $5(33.3 \%)$ & $6(10.0 \%)$ & \\
\hline $\mathrm{pN}$ & & & 0.785 \\
\hline N0 & $5(33.3 \%)$ & $16(26.7 \%)$ & \\
\hline N1 & $1(6.7 \%)$ & $13(21.7 \%)$ & \\
\hline N2 & $4(26.7 \%)$ & $13(21.7 \%)$ & \\
\hline N3a & $3(20.0 \%)$ & $15(25.0 \%)$ & \\
\hline N3b & $2(13.3 \%)$ & $3(5.0 \%)$ & \\
\hline pTNM stage & & & 0.346 \\
\hline I A & $3(20.0 \%)$ & $10(16.7 \%)$ & \\
\hline I B & $0(0.0 \%)$ & $5(8.3 \%)$ & \\
\hline II A & $1(6.7 \%)$ & $8(13.3 \%)$ & \\
\hline II B & $0(0.0 \%)$ & $4(6.7 \%)$ & \\
\hline III A & $4(26.7 \%)$ & $13(21.7 \%)$ & \\
\hline III B & $4(26.7 \%)$ & $12(20.0 \%)$ & \\
\hline III C & $3(20.0 \%)$ & $8(13.3 \%)$ & \\
\hline
\end{tabular}

Abbreviations: RG, domestic surgical robotic gastrectomy; LG, laparoscopic gastrectomy; BMI, body mass index; ASA, American Society of Anesthesiologists physical status classification; cTNM stage, clinical tumor node metastasis; pTNM stage, pathological tumor node metastasis.

Surgical factors and pathological parameters 
Surgical factors and pathological parameters are presented in Table 2. All operations were successfully completed, and no conversion or complications occurred during the operation. Postoperative pathological results showed that the pathological type of all gastric cancers was adenocarcinoma. The operative time was longer in the RG group than in the LG group (365.6 \pm $48.7 \mathrm{~min}$ vs. $298.6 \pm 70.9 \mathrm{~min}, \mathrm{P}<0.01)$. There was no significant difference in intraoperative blood loss, gastric resection, reconstruction, intraoperative blood transfusion or Chemoradiotherapy between the two groups $(\mathrm{P}>0.05)$. The histological type, Lauren's classification and proximal resection margin were not significantly different across the RG and LG groups. All tumor margins were negative, except in five specimens from the LG group. The $\mathrm{R} 0$ resection rates were similar between the two groups $(\mathrm{P}=0.238)$. And the harvested lymph nodes were also comparable between the RG group and the LG group [24.0 (16.0-36.0) vs. $20.0(10.0-47.0), \mathrm{P}=0.084]$.

Table 2 Surgical factors and pathological parameters

\begin{tabular}{llll}
\hline & $\begin{array}{c}\mathrm{RG} \\
(\mathrm{n}=15)\end{array}$ & $\begin{array}{c}\mathrm{LG} \\
(\mathrm{n}=19)\end{array}$ & $P$ value \\
\hline Operative time (min) & $365.6 \pm 48.7$ & $298.6 \pm 70.9$ & $<0.01$ \\
Intraoperative blood loss (ml) & $213.7 \pm 144.2$ & $219.2 \pm 155.6$ & 0.788 \\
Gastric resection & & & 0.753 \\
$\quad$ Distal & $13(86.7 \%)$ & $50(83.3 \%)$ & \\
$\quad$ Total & $2(13.3 \%)$ & $10(16.7 \%)$ & \\
Reconstruction & & & 0.753 \\
$\quad$ Billroth II & $13(86.7 \%)$ & $50(83.3 \%)$ & \\
$\quad$ Roux-en-Y & $2(13.3 \%)$ & $10(16.7 \%)$ & \\
Intraoperative blood transfusion & $4(26.7 \%)$ & $7(11.7 \%)$ & 0.142 \\
Chemoradiotherapy & $2(13.3 \%)$ & $19(31.7 \%)$ & 0.208 \\
Histological type & & & $>0.999$ \\
$\quad$ Differentiated & $5(26.7 \%)$ & $20(36.9 \%)$ & \\
$\quad$ Undifferentiated & $10(73.3 \%)$ & $40(63.1 \%)$ & \\
Lauren's classification & & & \\
$\quad$ Intestinal type & $7(46.7 \%)$ & $32(53.3 \%)$ & \\
\hline
\end{tabular}




\begin{tabular}{llll}
\hline \multicolumn{1}{c}{ Diffuse type } & $8(53.3 \%)$ & $24(40.0 \%)$ & \\
$\quad$ Mixed type & 0 & $4(6.7 \%)$ & \\
Proximal resection margin $(\mathrm{cm})$ & $3.8 \pm 2.9$ & $3.6 \pm 2.3$ & 0.879 \\
R0 resection rates & $15(100 \%)$ & $55(91.7 \%)$ & 0.247 \\
Harvested lymph nodes & $24.0(16.0-36.0)$ & $20.0(10.0-47.0)$ & 0.084 \\
\hline
\end{tabular}

Abbreviations: Billroth II, Billroth II gastrojejunostomy; Roux-en-Y, Roux-en-Y esophagojejunostomy.

\section{Postoperative outcomes and complications}

Postoperative outcomes and complications are shown in Table 3 and Table 4, respectively. There was no significant difference between the two groups in time of first flatus, time of liquid diet, time of abdominal drainage tube extraction, or hospital stay $(\mathrm{P}>0.05)$. According to the Clavien-Dindo classification system, there were 16 complications in the RG group, 6 in grade I, 10 in grade II, and no complications of grade III or above. Two patients had two or more complications. 68 complications were happened in the LG group, 22 in grade I, 44 in grade II, 1 in grade IIIa and 1 in grade IVa. Fourteen patients had two or more complications. The CCI was comparable between the two groups $(13.5 \pm 12.3$ vs. $13.7 \pm 14.6, \mathrm{P}=0.895)$. All patients were discharged smoothly. One patient of LG group died within 90 days after surgery due to poor physical condition and no further operation occurred.

Table 3 Postoperative outcomes

\begin{tabular}{llll}
\hline & $\begin{array}{c}\text { RG } \\
(\mathrm{n}=15)\end{array}$ & $\begin{array}{c}\text { LG } \\
(\mathrm{n}=19)\end{array}$ & $P$ value \\
\hline Time of first flatus (days) & $4.6 \pm 1.5$ & $4.2 \pm 1.7$ & 0.337 \\
Time of liquid diet (days) & $7.8 \pm 1.0$ & $7.7 \pm 2.2$ & 0.686 \\
Time of abdominal drainage tube & & & \\
extraction (days) & $8.0 \pm 0.9$ & $7.7 \pm 1.8$ & 0.655 \\
Hospital stay (days) & $11.0 \pm 2.8$ & $12.8 \pm 5.7$ & 0.189 \\
CCI & $13.5 \pm 12.3$ & $13.7 \pm 14.6$ & 0.895 \\
Death within 90 days after surgery & $0(0.0 \%)$ & $1(1.7 \%)$ & 0.615 \\
\hline
\end{tabular}

Abbreviations: CCI, comprehensive complications index. 
Table 4 Postoperative complications

\begin{tabular}{|c|c|c|c|}
\hline Clavien-Dindo grade & $\begin{array}{l}\mathrm{RG} \\
(\mathrm{n}=15)\end{array}$ & $\begin{array}{l}\mathrm{LG} \\
(\mathrm{n}=60)\end{array}$ & Treatment \\
\hline \multicolumn{4}{|l|}{$\mathrm{I}$} \\
\hline Fever & 0 & 9 & Antipyretics \\
\hline Dyspnea & 0 & 1 & Oxygen absorption \\
\hline Dizziness & 1 & 2 & Rest \\
\hline Stomachache & 2 & 2 & Analgesics \\
\hline Nausea or emesis & 0 & 4 & Antiemetics \\
\hline Edema & 1 & 0 & Diuretics \\
\hline Electrolyte disturbance & 2 & 4 & Electrolytes \\
\hline \multicolumn{4}{|l|}{ II } \\
\hline Hypertension & 0 & 1 & Hypotensive drugs \\
\hline Hypoproteinemia & 3 & 16 & Albumin \\
\hline Abnormal heart function & 1 & 1 & Cardiotonic drug \\
\hline Anastomotic hemorrhage & 0 & 4 & $\begin{array}{l}\text { Hemostatics and blood } \\
\text { transfusion }\end{array}$ \\
\hline Anemia & 0 & 2 & Blood transfusion \\
\hline Cough and Expectoration & 5 & 8 & Expectorant \\
\hline Pulmonary infection & 0 & 4 & Anti-infective drug \\
\hline Abnormal liver function & 0 & 3 & Hepatinica \\
\hline Fluctuation of blood pressure & 0 & 2 & Hyperensort or hypotensor \\
\hline Diarrhea & 0 & 1 & Antidiarrheic \\
\hline Digestive tract fistula & 0 & 1 & Conservative treatment \\
\hline Atrial fibrillation & 0 & 1 & Amiodarone \\
\hline Anal pendant expansion & 1 & 0 & Glycerine Enema \\
\hline \multicolumn{4}{|l|}{ III } \\
\hline \multicolumn{4}{|l|}{ IIIa } \\
\hline Intra-abdominal abscess & 0 & 1 & PTGD \\
\hline $\mathrm{IIIb}$ & 0 & 0 & \\
\hline \multicolumn{4}{|l|}{ IV } \\
\hline \multicolumn{4}{|l|}{ IV a } \\
\hline Pulmonary dysfunction & 0 & 1 & ICU management \\
\hline $\mathrm{IV} b$ & 0 & 0 & \\
\hline $\mathrm{V}$ & 0 & 0 & \\
\hline
\end{tabular}

Abbreviations: Clavien-Dindo grade, Clavien-Dindo Complications Classification System; PTGD, percutaneous transhepatic gallbladder drainage.

\section{Discussion}


Surgical robots with several advantages, such as three-dimensional imaging, high flexibility of instruments, hand tremor filtering, and humanized operating platform, are designed to overcome the limitations of laparoscopy. The research of surgical robots has gradually become a hot issues. New robotics technologies are emerging [15], such as the Invendoscopy E200 system, Flex ${ }^{\circledR}$ robot system, Senhance, Auris robotic endoscope system, and "Micro Hand S" surgical robot $[10,16]$. "Micro Hand $\mathrm{S}$ " surgical robot is a master-slave surgical robot developed by Central South University in conjunction with Tianjin University and Shandong WEGO Medical Robot Company. It consists of a surgeon console, a patient console, and accessories. The surgeon console includes a pedestal, column, armrest, main mechanical arm (left/right main mechanical arm), an image display device, and a control system. The patient console includes a base, lifting column, suspension arm, and an electrical control system of the slave mechanical arm (left/right slave mechanical arm). Accessories include 3D laparoscopic and camera systems, surgical instruments, sterile protective covers, and trocars. In March 2014, the "Micro Hand S" system began Phase I clinical trials at the Department of General Surgery, Third Xiangya Hospital, Central South University, and was registered at ClinicalTrials.gov (NCT02752698). The trial lasted 4 years and 10 months and ended in January 2019. In this study, 15 cases of robotic gastrectomy and 60 cases of laparoscopic gastrectomy were collected. The short-term outcomes of the two groups were compared to evaluate the safety and feasibility of "Micro Hand" surgical robotic gastrectomy.

The results of this study showed no statistically significant difference was observed in baseline characteristics between RG and LG groups, so clinical outcomes of these two groups could be compared. The surgical results showed that RG group had a longer operation time 
than LG group. It was because the operative time was increased due to inexperience and equipment installation. As technology advances and experience increases, we think the surgical time can be reduced [17]. The number of harvested lymph nodes in RG group was comparable with that in LG group $(\mathrm{P}=0.084)$, which may be related to the small sample size. There was no significant difference in $\mathrm{CCI}$ between the two groups $(\mathrm{P}=0.895)$. Although there was no significant difference in the $\mathrm{R} 0$ resection rate between the two groups $(\mathrm{P}=0.238)$, it was clear that five patients in the LG group had residual margin cancer, and no residual cancer occurred in the RG group. Three-dimensional field magnification and motion scaling of robotic surgery may be helpful in the precise removal of a mass. Perhaps a statistical difference can be found when a larger sample size was provided.

Furthermore, there was no significant difference in intraoperative blood loss, gastric resection, reconstruction, intraoperative complications, intraoperative blood transfusion, chemoradiotherapy, pathological type, Lauren's classification, proximal resection margin, time of first flatus, time of liquid diet, time of abdominal drainage tube extraction, duration of hospitalization or mortality within 90 days after surgery between the two groups. All postoperative complications were treated in a timely and effective manner. And all patients were discharged smoothly. No further operation occurred. The "Micro Hand S" system could perform gastrectomy and lymph node resection, and we think "Micro Hand S" surgical robotic gastrectomy is safe and feasible.

Of course, there are some limitations in our study. First, this study was retrospective and may be affected by selection bias. Second, the sample size was small witch could reduce the accuracy of surgical results. Third, the follow-up period was too short, and long-term 
follow-up and survival analysis is needed for the malignant tumor. Our medical center will be responsible for a multi-center, randomized, controlled clinical trial of "Micro Hand S" surgical robot, and the results will be reported later.

\section{Conclusion}

Ultimately, "Micro Hand S" surgical robotic gastrectomy is safe and feasible. And there will be one more choice of surgical procedure for patients in the future. We will continue evaluate the prognosis and quality of life of patients.

\section{Author Contributions}

Shaihong Zhu, Guohui Wang, Bo Yi, Zheng Li, Yong Liu and Yuanbing Yao contributed to the conception/design of the work. Yuanbing Yao and Yong Liu contributed to the acquisition of data. Yuanbing Yao and Yong Liu contributed to the analysis/interpretation of data. Yuanbing Yao drafted the manuscript. Shaihong Zhu, Guohui Wang, Bo Yi and Zheng Li revised the manuscript. All authors have approved the final version submitted and agreed to be accountable for all aspects of the work in ensuring that questions related to the accuracy or integrity of any part of the work are appropriately investigated and resolved.

\section{Funding information}

This study was supported by the National Key Research and Development Program of Special Project Foundation (Grant NO. 2017YFC0110402).

\section{Ethics approval and consent to participate}

This study was approved by the Institutional Review Board of the Third Xiangya Hospital. All patients signed a written informed consent. Data collection and analysis were performed in compliance with the Helsinki Declaration of 1975.

\section{Conflict of Interest}

The authors declare that they have no competing interests and agree to publish this article.

\section{Author details}

1 Department of General Surgery, Third Xiangya Hospital, Central South University, 138 Tongzipo Street, Changsha 410013, Hunan, China

\section{References}

[1] Sitarz R, Skierucha M, Mielko J, Offerhaus GJA, Maciejewski R, Polkowski WP. Gastric cancer: epidemiology, prevention, classification, and treatment. Cancer Manag. Res. 2018;10:239-48. 
[2] Chen WQ, Sun KX, Zheng RS, Zeng HM, Zhang SW, Xia CF, et al. Cancer incidence and mortality in China, 2014. Chin. J. Cancer Res. 2018;30(1):1-12.

[3] Liu HB, Wang WJ, Li HT, Han XP, Su L, Wei DW, et al. Robotic versus conventional laparoscopic gastrectomy for gastric cancer: A retrospective cohort study. Int. J. Surg. 2018;55:15-23.

[4] Lu J, Zheng HL, Li P, Xie JW, Wang JB, Lin JX, et al. A Propensity Score-Matched Comparison of Robotic Versus Laparoscopic Gastrectomy for Gastric Cancer: Oncological, Cost, and Surgical Stress Analysis. J. Gastrointest. Surg. 2018;22(7):1152-62.

[5] Suda K, Man-i M, Ishida Y, Kawamura Y, Satoh S, Uyama I. Potential advantages of robotic radical gastrectomy for gastric adenocarcinoma in comparison with conventional laparoscopic approach: a single institutional retrospective comparative cohort study. Surg. Endosc. 2015;29(3):673-85.

[6] Hashizume M, Shimada M, Tomikawa M, Ikeda Y, Takahashi I, Abe R, et al. Early experiences of endoscopic procedures in general surgery assisted by a computer-enhanced surgical system. Surg. Endosc. 2002;16(8):1187-91.

[7] Song J, Kang WH, Oh SJ, Hyung WJ, Choi SH, Noh SH. Role of robotic gastrectomy using da Vinci system compared with laparoscopic gastrectomy: initial experience of 20 consecutive cases. Surg. Endosc. 2009;23(6):1204-11.

[8] Hyun MH, Lee CH, Kwon YJ, Cho SI, Jang YJ, Kim DH, et al. Robot Versus Laparoscopic Gastrectomy for Cancer by an Experienced Surgeon: Comparisons of Surgery, Complications, and Surgical Stress. Ann. Surg. Oncol. 2013;20(4):1258-65.

[9] Lee S, Kim JK, Kim YN, Jang DS, Kim YM, Son T, et al. Safety and feasibility of reduced-port robotic distal gastrectomy for gastric cancer: a phase I/II clinical trial. Surg. Endosc. 2017;31(10):4002-9.

[10] Yi B, Wang GH, Li JM, Jiang J, Son Z, Su H, et al. The first clinical use of domestically produced Chinese minimally invasive surgical robot system "Micro Hand S". Surg. Endosc. 2016;30(6):2649-55.

[11] Japanese Gastric Canc A. Japanese gastric cancer treatment guidelines 2014 (ver. 4). Gastric Cancer 2017;20(1):1-19.

[12] In H, Solsky I, Palis B, Langdon-Embry M, Ajani J , Sano T. Validation of the 8th Edition of the AJCC TNM Staging System for Gastric Cancer using the National Cancer Database. Ann. Surg. Oncol. 2017;24(12):3683-91.

[13] Slankamenac K, Graf R, Barkun J, Puhan MA, Clavien PA. The Comprehensive Complication Index A Novel Continuous Scale to Measure Surgical Morbidity. Ann. Surg. 2013;258(1):1-7.

[14] Dindo D, Demartines N, Clavien PA. Classification of surgical complications - A new proposal with evaluation in a cohort of 6336 patients and results of a survey. Ann. Surg. 2004;240(2):205-13.

[15] Peters BS, Armijo PR, Krause C, Choudhury SA, Oleynikov D. Review of emerging surgical robotic technology. Surg. Endosc. 2018;32(4):1636-55.

[16] Yi B, Wang GH, Li JM, Jiang J, Son Z, Su H, et al. Domestically produced Chinese minimally invasive surgical robot system "Micro Hand S" is applied to clinical surgery preliminarily in China. Surg. Endosc. 2017;31(1):487-93.

[17] Kim YW, Reim D, Park JY, Eom BW, Kook MC, Ryu KW, et al. Role of robot-assisted distal gastrectomy compared to laparoscopy-assisted distal gastrectomy in suprapancreatic nodal dissection for gastric cancer. Surg. Endosc. 2016;30(4):1547-52. 
Figures
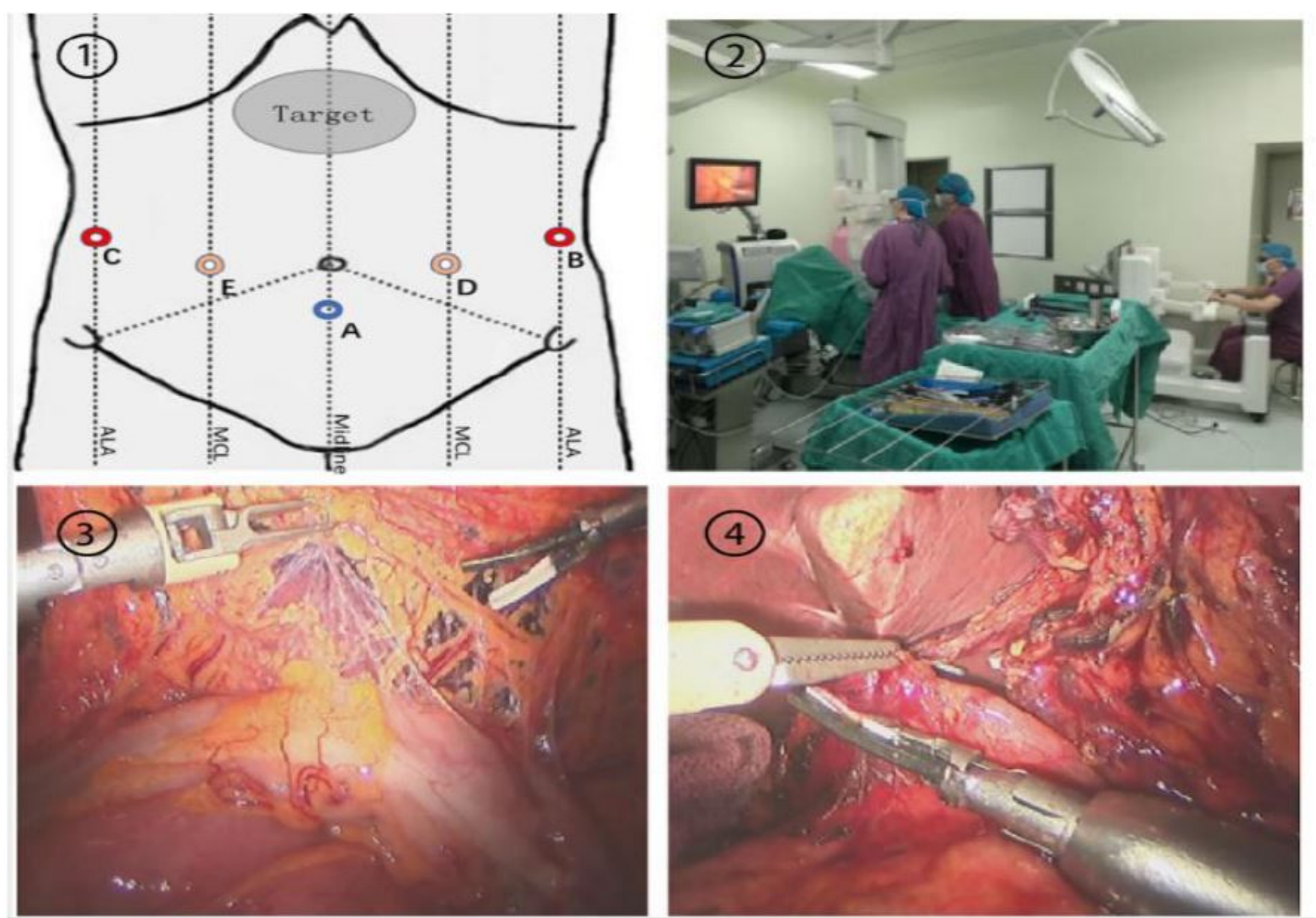

\section{Figure 1}

Trocar placement and operation of the domestic robot. $\otimes$ Trocar position. $\otimes$ The operating room. $\nabla$ Separating gastrocolic ligament. $\otimes$ Freeing common hepatic artery. 